\title{
«Sulle orme di Chateaubriand e di Stendhal». Malaparte e la Francia
}

Damiano Sinfonico

\section{(2) OpenEdition}

1 Journals

\section{Edizione digitale}

URL: http://journals.openedition.org/studifrancesi/5502

DOI: $10.4000 /$ studifrancesi.5502

ISSN: 2421-5856

\section{Editore}

Rosenberg \& Sellier

\section{Edizione cartacea}

Data di pubblicazione: 1 septembre 2011

Paginazione: 356-365

ISSN: 0039-2944

\section{Notizia bibliografica digitale}

Damiano Sinfonico, ««Sulle orme di Chateaubriand e di Stendhal». Malaparte e la Francia», Studi

Francesi [Online], 164 (LV | II) | 2011, online dal 30 novembre 2015, consultato il 09 janvier 2021. URL: http://journals.openedition.org/studifrancesi/5502 ; DOI: https://doi.org/10.4000/studifrancesi.5502

\section{(c) (1)}

Studi Francesi è distribuita con Licenza Creative Commons Attribuzione - Non commerciale - Non opere derivate 4.0 Internazionale. 


\section{«Sulle orme di Chateaubriand e di Stendhal». Malaparte e la Francia}

\section{Malaparte in Francia}

Quando nelle prime pagine di La pelle Malaparte presenta il suo amico e compagno di viaggio, il colonnello Jack Hamilton, compie una lenta discesa verso una realtà che sembra intrecciarsi e spaccare quella che le si oppone: nell'autunno del 1943, nella Napoli appena liberata dove i bambini e le bambine vengono venduti come in un mercato ai soldati americani, Jack si muove stupito in una città straniera e strana, e progressivamente Malaparte rivela al lettore l'intima natura di questo americano nutrito di letteratura, da quelle classiche a quella francese e americana. Orgoglioso del suo francese appreso su La Fontaine e dalla sua portinaia di Rue Vaugirard, il colonnello sa citare a memoria Omero o appartarsi con in mano una copia di Pindaro, fermarsi a parlare di Winckelmann e a vagheggiare una «Grecia francese, una Grecia del diciottesimo secolo» ${ }^{1}$, mentre Malaparte pesca dal Seicento francese un episodio che per la sua studiata frivolezza diventa non un contraltare alla tragedia europea, bensì un punto focale attraverso cui osservarla:

...O gli accadeva di confondere la «foglia parlante» di Madame de Sévigné con un animale parlante di La Fontaine.

«Non era un animale» gli dicevo «era una foglia, una foglia d'albero.»

E gli citavo il passaggio di quella lettera, nella quale Madame de Sévigné scriveva che avrebbe desiderato ci fosse, nel parco del suo castello dei Rochers, in Bretagna, una foglia parlante.

«Mais cela c'est absurde» diceva Jack «une feuille qui parle! Un animal ça se comprend, mais une feuille!»

«Per capire l'Europa» gli dicevo «la ragione cartesiana non serve a nulla. L'Europa è un paese misterioso, pieno di segreti inviolabili»².

Il passo, (quasi) mai citato negli studi malapartiani, ci sembra un ottimo punto di partenza per comprendere non tanto o non solo quanto la letteratura francese abbia attirato una continua attenzione da parte dello scrittore pratese, ma anche e soprattutto per capire come la Francia abbia significato qualcosa, più nello spazio interiore dello scrittore (e della sua scrittura) che come luogo fisico, nel costruire un personale mito di Parigi che certamente sostituisce quello di Roma e diventa una ideale, simbolica patria della civiltà e delle lettere nel momento della loro distruzione.

Prima di procedere ad un'analisi puntuale di questo tema nelle opere, può essere vantaggioso ricordare alcune tappe biografiche che segnano il legame duraturo tra lo scrittore e la Francia, iniziato durante l'adolescenza, quando il giovane Suckert si arruola nella Legione Garibaldina e a tempi alterni sarà sul fronte francese, per poi

(1) C. Malaparte, Opere scelte, a cura di L. MaRTELLINI e con una testimonianza di G. VigoreLLI, Milano, Mondadori (Meridiani), 1997, p. 979. (Da qui in poi citeremo da questo volume abbreviando con K per Kaputt e P per La pelle).

(2) P 980. 
assistere come ufficiale di ordinanza alla conferenza di Versailles. Ma il primo soggiorno stabile nella capitale avverrà solo nel biennio 1931-1933, quando Malaparte lascia l'Italia dopo essere stato sciolto dall'incarico di direttore della «Stampa»: a Parigi pubblica per Grasset Technique du coup d'État, il libro che gli dà grande fama internazionale, proibito in Italia e Germania, e pubblicato in Italia solo nel 1948 in una versione rimaneggiata rispetto a quella francese. Al biennio 1947-1949 risale il secondo soggiorno parigino, nel momento in cui l'ostilità nei confronti del personaggio si fa acre e lo scrittore si illude di ritrovare oltralpe un clima favorevole: a Parigi mette in scena due opere teatrali scritte direttamente in francese, Du côté de chez Proust e Das Kapital, cui però non arride il successo, mentre in contemporanea vengono pubblicate l'edizione italiana e francese di La pelle, tradotta da René Novella in concomitanza con la stesura dell'opera; di questa seconda esperienza Malaparte prende nota nel Diario di uno straniero a Parigi, pubblicato postumo da Vallecchi nel 1966.

A questa passione malapartiana per la Francia fa da riflesso quella francese per Malaparte: è nota la straordinaria fortuna che la sua opera ha avuto in Francia, dove secondo un recente spoglio 3 si contano a oggi ben 48 edizioni, che fanno di Malaparte uno degli autori italiani più tradotti, insieme a Moravia, Calvino, Buzzati e Sciascia: un "risultato" che inizia sotto buoni auspici se già nel 1927 la sua prima pubblicazione francese, L'Italie contre l'Europe, portava la prefazione di Benjamin Crémieux, per arrivare al recente riconoscimento di due illustri académiciens come André Gluck$\mathrm{smann}^{4}$ e Marc Fumaroli ${ }^{5}$. Ciononostante anche in Francia non sempre l'accoglienza è stata pacifica (si ricordi la freddezza di intellettuali come Sartre e Camus) e alcune riserve anche aspre sul personaggio non sono mancate ${ }^{6}$, mentre numerose sono le recensioni che accolgono Kaputt e La pelle come veri capolavori: tra queste, una recensione è singolare perché sembra suggerire il segreto del successo francese di Kaputt, ovvero l'insistente ritorno su una francesità come dato culturale ed emotivo attorno a cui si aggrega l'itinerante esperienza bellica ( «Songez que ce poète, cet homme civilisé, est européen qui a longtemps vécu en France, qui à tout instant rêve du ciel

(3) D. Valin (a cura di), Bibliographie des traductions françaises de la littérature italienne des 20 e 21e siècles, in «Chroniques italiennes», n. 66/67. Si contano ben sette edizioni di Kaputt (tra il 1946 e il 2006), cinque di La pelle (tra il 1949 e il 2008), cinque di Il sole è cieco (tra il 1958 e il 2010, un caso anomalo visto che l'unica edizione italiana risale al 1957 e spiegabile con il fatto che il romanzo si svolge sulle Alpi francesi all'inizio della guerra). Si rimanda, per la fortunata ricezione straniera in generale e francese in particolare, anche al catalogo della mostra Il boulevard delle diversità. Da Parigi a Pechino, uno scrittore intorno al mondo, a cura di M. Grassi e F. Goti, Biblioteca comunale Alessandro Lazzerini, Prato, 2007.

(4) Cfr. A. Cazzullo, Un grande emarginato. Glucksmann: "Ha guardato il male in volto", in «La Stampa», 3 settembre 1998, dove l'accademico francese rende omaggio a Malaparte e a Kaputt in particolare, considerato una testimonianza lucida e impegnata della barbarie della guerra, poiché Malaparte avrebbe saputo guardare il male in volto come pochi: «È questo il momento di chiarezza che fa il grande intellettuale. Perché l'intellettuale non è il buon pastore, il taumaturgo che porge le chiavi del paradiso; ma la sentinella del Nulla, che osa dire quel che non si vuol sentire».
(5) Cfr. M. Fumaroli, La grande rivolta del moderno, in «Repubblica», 24 aprile 2009. L'articolo è una recensione all'ultimo libro di Kundera, Un Incontro (Adelphi, 2009), il quale dedica un capitolo a Malaparte, elogiato come uno dei massimi scrittori del Novecento, partecipe del rinnovamento della forma romanzo, pienamente inserito in quel filone modernista che nel corso del secolo ha messo in discussione il genere e prodotto nuovi frutti. L'apprezzamento entusiasta di Fumaroli eleva Malaparte al pantheon dei grandi scrittori europei del secolo, arrivando a scrivere che Kaputt e La pelle, «lontanissimi dal volgare reportage, sono opere d'arte, scritte dal punto di vista superiore del poeta, sfolgoranti delle verità dolorose e contraddittorie che sono esclusivo appannaggio della poesia e del grande romanzo. [...] Malaparte ha innalzato la sua prosa alle altezze del Waste Land di Thomas Stearns Eliot».

(6) Per questo argomento si rimanda a J.-C. THIRIET, Paris la haine, in Curzio Malaparte il narratore, il politologo, il cittadino di Prato e dell'Europa. Atti del Convegno di Prato 1998, a cura di R. Barilli e V. BARONCELli, CUEN, Napoli, 2000, pp. 314-325; e a A. SARRABAYROUSE, Malaparte et l'attrait contrasté de la France, in «Chroniques italiennes», n. 44 (4/1995). 
de Paris... qui sur le front russe rêve d'un paysage de Monet, d'une nature morte de Braque, d'un azur de Chagall; pense à l'univers proustien et se répète des vers d'Apollinaire...» ${ }^{7}$ ) e la mancanza di un equivalente nelle lettere francesi:

ce conte de mille et une nuits d'épouvante et d'horreur ... est une œuvre qui, par la diversité, l'accent et l'éclat, n'a pas encore d'équivalent en France. Non seulement son intérêt historique et dramatique demeure inégalé, mais elle ressortit à un genre dont il semble que certaines littératures étrangères gardent la maitrise. ... De ces livres de haute culture, de savoir universel émanant d'écrivains ayant parcouru le monde, familiers avec Rome, avec Londres, avec Madrid, avec Paris, avec la culture antique et la culture moderne, et pour qui un lever de soleil sur le front russe appelle instantanément dans la mémoire un passage de l'Anabase de Xénophon. Écrivains puissants et raffinés dont chaque page est celle d'un peintre, d'un artiste, d'un Européen ${ }^{8}$.

Su questo tenore la stampa francese ha continuato a riservare, nel corso dei decenni, un'attenzione particolare a Malaparte, ricordato nel trentennale della morte come «L'Européen exemplaire» ${ }^{9}$ e «le plus génial pasticheur de notre temps» ${ }^{10}$ e celebrato su «Le Figaro» nel centenario della nascita. Perché questa fortuna all'estero mentre in Italia manca un riconoscimento pacifico? Spezzando la questione in due segmenti, il mancato riconoscimento in patria può essere ricondotto a ragioni storiche, cioè il compromesso ambiguo e irritante di Malaparte con il regime, per le quali «la letteratura italiana sembra macchiarsi di un eccesso di legittima difesa» ${ }^{11}$, mentre al perché del successo d'oltralpe sembra rispondere l'analisi di Mario Isnenghi, che vede Kaputt destinato a un «pubblico sovranazionale, europeo» ${ }^{12}$ per il suo «sguardo sovranazionale, d'insieme, non di parte» ${ }^{13}$.

(7) P. Loewel, Kaputt par Curzio Malaparte, in «L'Aurore», 11 settembre 1946 e poi in E. RONCHI SuCKERT, Malaparte, Ponte alle Grazie, Firenze, 1993, vol. VII (1946-1947), pp. 141-143.

(8) Ibid.

(9) B. Poirot-Delpech, L’Européen exemplaire, in «Le Monde», 24 luglio 1987.

(10) J.-N. SCHIFANO, Un chien comme moi, in «Le Monde», 24 luglio 1987.

(11) A. DEBENEDETTI, Malaparte kaputt?, in «Corriere della Sera», 19 settembre 1992. In chiusura d'articolo Malaparte viene presentato come «uno scrittore forse troppo scopertamente furibondo, turgido, fantasiosamente bugiardo ma anche affascinante, geniale, privo di scrupoli ma anche di timidezze». E interessante uno spoglio per studiare la posizione di Malaparte nelle recenti storie letterarie italiane, dove si passa dal silenzio o un'estrema marginalità (SEGRE-MARTIGNONI per Mondadori, 2000), dal riconoscimento solo di opere giovanili e movimentate (AsOR Rosa per Einaudi, 2009) al tentativo di un medaglione che ne metta in luce i difetti come i meriti (BORSELLINO-PEDULLÀ per Motta, 1999, e BÀrBeri SQuAROTTI per UTET, 1996), tra i quali il più volte riconosciuto talento di prosatore, a chi vede in Malaparte uno scrittore centrale ed emblematico, autore di opere di grande rilievo (Malato per Salerno Ed., 2000). Rimane valida l'indicazione di Montale che nel necrologio riconduceva le diverse reazioni a «quel sentimento "ambivalente" che i veri scrittori lasciano quando li sentiamo diversi da noi» (E. MonTALE, L'arcitaliano, in «Corriere della Sera», 20 luglio 1957 e poi in Il secondo mestiere. Prose 1920-1979, Milano, Mondadori, 1996, pp. 2062-2068, a p. 2062). Tra gli interventi più recenti si ricordano le parole di Maurizio Serra, che ha scritto: «Vi sono molte ragioni, tutte legittime, per non amare Malaparte uomo, scrittore e personaggio. Ma nessuna, a nostro avviso, per negargli un posto di primo piano tra gli interpreti più singolari di un Ventesimo secolo le cui inquietudini si prolungano nel nostro» (M. SERRA, Malaparte: volti e maschere di un esteta armato, introduzione al catalogo della mostra Malaparte arcitaliano nel mondo, Biblioteca di Via Senato, Milano, 2010, pp. 9-13).

(12) M. IsNenghI, Introduzione a C. Malaparte, Kaputt, Mondadori, Milano, 1979, p. 12.

(13) Ibid. 


\section{Parigi nella scrittura di Malaparte}

Se il primo capitolo di Kaputt si intitola «Le côté de Guermantes», ogni lettore è autorizzato a leggere l'intero libro sotto il segno di Proust: lo ha fatto eccellentemente Jean-Claude Thiriet ${ }^{14}$, che in un suo intervento ha messo magistralmente in luce $\mathrm{i}$ rapporti tra Malaparte e Proust, dopo aver dedicato qualche pagina alla presenza e al significato della lingua francese nel libro: ci sembra utile proseguire in questa direzione per dare una lettura integrale e d'insieme in cui si combinino tutti gli aspetti francesizzanti dell'opera malapartiana, per ritrovare al centro o dietro la tessitura un mito di Parigi, se così possiamo chiamarlo, attraverso il quale Malaparte compie un'operazione delicata di messa in discussione della letteratura e dei suoi mezzi: la modernità della sua scrittura passa per la mancanza di una struttura lineare in Kaputt, per la dissoluzione delle forme tradizionali in un ibrido di generi, di lingue e di stili che restituisce un volto vero alla catastrofe della guerra, tolta la maschera della retorica. Se la forma romanzo non è ammissibile, perché troppo limitata a una certa unità di azione, e rimane la sua moltiplicazione in un gioco di specchi e di cornici, di segmenti narrativi che si interrompono e vengono ripresi, intrecciati, ripetuti, tentacolarmente dipanati su uno spazio grande come mezza Europa, allora la forma romanzo diventa un archetipo fluido in cui ciò che è leggero si lascia trascinare e ciò che è pesante rimane al fondo, ma all'origine di questi smottamenti ci deve essere un centro, non unificatore ma almeno di riferimento: un luogo fisico a cui si allude nel momento in cui lo si nega, che si tratteggia con amore quando viene evocato, si riverbera quasi in ogni pagina grazie all'esportazione della sua lingua che per la sua diffusione irraggia grazia e civiltà: Parigi è non solo a livello tematico, come ha individuato Thiriet, il simbolo di un un'Europa civile minacciata dalla barbarie nazista, bensì è anche un elemento strutturale che attraversa ed esercita pressione su tutto il libro, è il solo elemento aggregante (e non il narratore-protagonista) attraverso cui l'intera materia trova un centro fisico, spaziale assieme alla coscienza di una deriva irreversibile. Questo centro è vitale per un mondo assurdamente decentrato, dove la crudeltà è la stessa in ogni luogo, per cui narrarne un segmento significa trovare l'universale nel particolare, ma perdere il tessuto invisibile che collega tutti gli eventi: abbandonata l'unità romanzesca, il caos del mondo frammentato trova il suo volto più vero in una ferocia che deresponsabilizza il singolo e si riflette all'infinito in un presente perpetuo e liquido.

Parigi non è solo un'ideale patria delle lettere ormai possibile solo nel mito, ma rappresenta soprattutto la ricerca disperata di un centro: Proust e gli altri innumerevoli scrittori (e pittori) francesi citati in Kaputt non possono essere valutati solo secondo il criterio delle influenze, bensì sono i nodi di una rete che vuole essere segno di una faticosa unità ricomposta, di una realtà a cui la letteratura rimanda per indicare la propria legittimità e la volontà di continuità pur nella rottura: la nuova forma "arciromanzo" (per usare l'espressione di Kundera) per Malaparte significa l'impossibilità di una forma che non sia riciclo e collage di pezzi precedenti, come delle toppe su una realtà in disfacimento. Parigi si trova al centro di questa costellazione come un eden precario sulle sabbie mobili del ricordo e della guerra; è doppiamente significativo che in Kaputt nessuna scena si svolga a Parigi, presente solo nel ricordo e nel sogno, e che in La pelle il compagno di viaggio Jack sia connotato di un passato parigino.

Nel primo capitolo di Kaputt una Parigi fin de siècle è intimamente legata alla

(14) J.-C. THIRIET, L'influenza perenne: Malaparte 'francese', in Malaparte scrittore d'Europa. Atti del convegno (Prato 1987) e altri contributi, a cura di
G. Grana e V. Baroncelli, Prato, Marzorati, 1991, pp. $135-145$. 
gioventù dell'ottuagenario principe Eugenio di Svezia, aristocratico che aveva trascorso alcuni anni fra i quartieri artistici della capitale francese come pittore, allievo di Puvis de Chavanne e di Bonnat. Malaparte associa e poi confonde il principe con i personaggi di Proust, quasi sul punto di chiedergli se avesse mai conosciuto il Duca e Madame de Guermantes, quando il principe prende la parola e

si mise a parlar di Parigi, come se Parigi, ai suoi occhi di pittore, fosse soltanto un colore, la memoria, la nostalgia di un colore ... Forse Parigi non era per lui che un suono: i suoi ricordi visivi, le immagini dei suoi giovani anni parigini, spogliati d'ogni qualità sonora, vivevano nella sua memoria di per se stessi, si muovevano, si illuminavano, volavano via «comme les monstres ailés de la préhistoire». Le mute immagini di quella sua giovane, remota Parigi, gli crollavano davanti agli occhi senza rumore, senza che il crollo di quel felice mondo della sua giovinezza «ternisse, de la vulgarité d'aucun bruit, la chasteté du silence».

$[\ldots]$

Sulle immagini della remota Parigi di Madame de Guermantes e del Principe Eugenio, si sovrapponevano a poco a poco, davanti ai miei occhi, le dolorose e care immagini di una Parigi più giovane, più torbida, più inquieta, più triste, forse. Come i visi dei passanti che affiorano dalla nebbia di là dai vetri di un caffè, io vedevo affacciarsi alla memoria i volti di Albertine, di Odette, di Robert de Saint Loup, le ombre degli adolescenti che s'intravedono dietro le spalle di Swann e di M. De Charlus, le fronti segnate dall'alcool, dall'insonnia, e dalla sensualità, dei personaggi di Apollinaire, di Matisse, di Picasso, di Hemingway, gli spettri azzurri e grigi di Paul Éluard. «Ho visto i soldati tedeschi in tutte le città d'Europa» dissi, «ma non voglio vederli a Parigi».

Il Principe Eugenio reclinò il capo sul petto, e disse con voce lontana: «Paris, hélas!» ${ }^{15}$.

Questa meravigliosa pagina di sapore proustiano, segno, come tanto Malaparte, di una «raffinatezza ed eleganza tipiche del decadentismo europeo» ${ }^{16}$, oltre a legare tematicamente Parigi a un'epoca felice e civile non più possibile nel presente, istituisce il nesso Parigi-giovinezza che ritornerà nell'altra pagina in cui ricorre l'evocazione in sogno di una Parigi vissuta in prima persona dal protagonista: al capitolo sesto, una notte nel villaggio romeno di Jassy, il protagonista sogna di ritrovarsi a Parigi $(\ll \mathrm{Ri}-$ conobbi sui tetti il cielo di Parigi») e di dirigersi verso casa propria. Ma ad aprirgli la porta è un Malaparte più giovane di dieci anni che non riconosce il nuovo arrivato e lo squadra con sospetto, così come gli amici riuniti nella casa (tra cui Giraudoux, Pirandello, Malraux, Guehénno...):

Tutti i miei amici son lì, davanti a me, seduti in silenzio e alcuni di loro son morti, hanno il viso pallido e gli occhi spenti. Forse son rimasti lì ad aspettarmi tutti quegli anni, e non mi riconoscono. ... mi affaccio alla finestra, e vedo i ponti di Parigi... ${ }^{17}$

C'è qualcosa di tragico in questo mancato riconoscimento, nello stravolgimento di un'identità che non è più riconoscibile mentre i personaggi rimangono fissi nella memoria, in un luogo impenetrabile, non più raggiungibile se non al prezzo di sentirvisi estranei: tema tradizionale, certamente, che qui segnala anche in una dimensione più ampia una deriva a cui la stessa identità personale non si sottrae, mentre il ritorno sempre doloroso se non impossibile sancisce l'impossibile rinascita della Parigi di un tempo, affidata alla memoria o al sogno, a una realtà quindi straniante ma che riposa nell'universo interiore del protagonista, ne investe il passato e ne plasma i sogni, ne

(15) K 462-463.

(16) L. MARTELLINI, Introduzione a C. Malaparte,
Opere scelte, cit., p. XLIV.

(17) K 558. 
costituisce il cuore pulsante e l'origine di una scrittura insidiata dalla barbarie che vuole raccontare.

In La pelle Malaparte dedica un paragrafo alla Parigi da cui proviene l'amico americano Jack. È interessante la densità di riferimenti letterari puntuali, il poetico pastiche linguistico con cui rende l'atmosfera cosmopolita e decadente di una città pullulante di giovani sradicati alla ricerca di qualcosa:

Jack non era di quegli Américains de Paris che s'incontrano ad ogni pagina di The sun also rises di Hemingway, che intorno al 1925 frequentavano il Select di Montparnasse, che disdegnavano i tè di Ford Madox Ford e la libreria di Sylvia Beach: e dei quali Sinclair Lewis, a proposito di certi personaggi di Eleanor Green, dice che erano «come i profughi intellettuali della Rive Gauche verso il 1925, come T. S. Eliot, Ezra Pound, o Isadora Duncan, iridescent flies caught in the black web of an ancient and amoral european culture». Jack non era neppure di quei giovani decadenti d'oltre Atlantico raccolti intorno alla rivista americana «Transition», che si stampava a Parigi verso il 1925. No, Jack non era un déraciné né un decadente: era un americano innamorato dell'Europa ${ }^{18}$.

Pur staccando Jack da un fondale quasi di corruzione, portata da quegli stessi americani che hanno liberato l'Italia, Malaparte mostra il potere seduttivo di una capitale del mondo, città incantatrice da cui Jack esce con un amore puro per l'Europa e che offre al narratore una materia in cui il canto delle sirene fa luccicare di una vitalità nuova e postrema questa culla della civiltà: se la fine appare imminente, Jack può muoversi in tutto il libro preservando un amore puro per l'Europa perché porta con sé l'anima di Parigi in un viaggio attraverso le vergogne dell'Italia liberata.

Al decimo capitolo Parigi significativamente tornerà quando i liberatori sono alle porte di Roma: in questo capitolo asperrimo nei confronti dell'Italia umiliata, dove le rovine romane sono derise dai liberatori mentre Malaparte cerca di illustrarle, i soldati francesi e lo stesso narratore rimpiangono di veder liberata Roma prima di Parigi; e mentre si avvicinano all'Urbe commentano l'atmosfera che ha qualcosa di familiare, un «air français qu'y ont mis le Poussin e Claude Lorrain» ${ }^{19}$, mentre il generale tira fuori da una tasca le Promenades dans Rome di Stendhal. Ormai Roma si presenta come un'emanazione di Parigi, «una capitale di provincia $\gg^{20}$ alla periferia dell'Europa civile, una città che si tiene viva solo per l'assidua pratica del pettegolezzo in una vita mondana vuota, quale stendhalianamente viene descritta nell'ultima sezione di Kaputt: la frivolezza è rimasta l'unica molla vitale delle sue piccoli corti, e il passato splendore può rinnovarsi in modo effimero sulla bocca di aristocratici inerti, come avviene per il giovane Anthony Eden, che dopo aver «vivamente eccitato la società romana $>^{21}$ riparte all'improvviso per Londra e la sua favola si chiude con un eloquente scambio di battute:

«Che straordinaria città è Roma!» disse Lord Perth «L'eternità vi si respira nell'aria. Tutto diventa materia di leggenda, anche il pettegolezzo mondano. Ecco dunque Sir Anthony Eden entrato nella leggenda. Il lui a suffi d'un séjour d'une semaine dans la Ville Eternelle, pour entrer dans l'éternité».

«Oui, mais il en est sorti bien vite, le malin!» disse Jane ${ }^{22}$.

(18) P 980-981.

(19) P 1259.

(20) K 385
(21) K 388

(22) K 389-390. 
Sintomatico è anche che per descrivere la vita romana Malaparte ricorra a mezzi francesi: non solo l'uso della lingua nel passo ora citato, ma la Città Eterna ormai è riconoscibile solo tramite le descrizioni che ne hanno fatto gli scrittori francesi, nella direzione di una francesità che si costituisce come la mappatura puntuale di un mondo satellite e assorbito. Se nella scoperta di Roma i due generali francesi Guillaume e Juin e il diplomatico inglese Anthony Eden si muovono «sulle orme di Chateaubriand e di Stendhal $\gg^{23}$, se la corte di Isabella Colonna assomiglia tanto a quella di una Sanseverina per i dinamismi che la animano e per «i soliti beaux esprits di Roma, che eran pur sempre quelli di cui parla Stendhal $\gg^{24}$, pagine bellissime sono dedicate alla contessa Edda Ciano, nuova Madame Bovary, «donna mortalmente triste ${ }^{25}$ protagonista suo malgrado di una «histoire romanesque ${ }^{26}$. Bastano queste citazioni per comprendere che se tematicamente Roma è disprezzata, anche per un rovesciamento della retorica fascista, nel confronto con Parigi, quest'ultima ricopre lungo tutto Kaputt e La pelle una funzione di primaria importanza volta a garantire un senso e un'unità all'odissea del protagonista nell'Europa devastata dalla guerra e nell'Italia umiliata.

\section{Il francese e ifrancesi nella scrittura di Malaparte}

In un articolo pubblicato nel 1934, Crisi del romanzo francese $e^{27}$, Malaparte fa partire la sua riflessione dal recente successo di Céline, e concorda con l'amico ed editore Max Dorian per il quale «se Balzac tornasse al mondo, rimarrebbe disgustato dalla perfezione alla quale è giunto il nostro romanzo», riconosce a Proust un grande genio che però invece di avere aperto nuove porte le ha chiuse tutte, e lamenta che «questa arte è diventata una formula», per concludere con la domanda se il romanzo «il rimedio al proprio male dovrà cercarlo in se stesso, nella propria perfezione, o al di fuori», considerato questo «il solito dilemma che si pone a una tradizione letteraria nel momento del suo estremo splendore». Sei anni più tardi, in un articolo dal titolo surreale di Cadaveri squisiti ${ }^{28}$, Malaparte scriverà che «la nostra stessa opera di scrittori, insomma, non è che un detrito di un mondo formalmente e moralmente in rovina»: la gestazione di Kaputt da qui a pochi anni riesce a rispecchiare la rovina della civiltà europea e allo stesso tempo confermare l'esigenza di reperire nuovi mezzi fin nella lingua stessa.

Se Kaputt non ha un andamento lineare e progressivo, anche la sua lingua si disperde in mille rivoli per un pastiche linguistico che mostra in superficie la polverizzazione delle sue strutture: mancante di un centro, anche la molteplicità delle lingue veicola la molteplicità di angolazioni da cui la realtà può essere osservata, gli infiniti binari su cui corrono i destini umani; c'è una vertigine per l'infinito che porterebbe il narratore a impadronirsi di tutte le lingue parlate, renderlo un domatore della babele linguistica per poter superare l'attrito tra la parzialità di una lingua e l'apertura inesauribile della realtà. Solo così poteva essere reso il mosaico europeo di culture che vede al centro quella francese: la lingua francese, come è stato più volte osservato,

(23) K 387

(24) K 390

(25) K 309

(26) K 312

(27) C. Malaparte, Crisi del romanzo francese, in «Corriere della Sera», 30 novembre 1934, e ora in
E. Ronchi Suckert, Malaparte, Ponte alle Grazie, Firenze, 1992, vol. III (1932-36), p. 550 e ss.

(28) C. Malaparte, Cadaveri squisiti, in «Prospettive», n. 5, 15 luglio 1940 e poi in E. RoNCHI SuCKERT, op. cit. vol. V (1940-41), pp. 267-271. 
ricorre in quasi tutti i dialoghi con interlocutori colti e raffinati, quasi a cementare un'ideologia o una coscienza di classe comune che supera le barriere nazionali e si identifica in quella civiltà delle bonséances. Non è solo una scelta mimetica, si tratta di una calcolata scelta poetica che vuole far saltare il legame intimo tra l'artista e la sua lingua («la lingua ... è cosa alla quale uno scrittore è intimamente legato [...] Non son forse, il mondo poetico e il linguaggio di uno scrittore, una sola cosa? $\left.\rangle^{29}\right)$, renderla inadeguata perché il mondo è molto più complesso di un incasellamento linguistico, perché solo la proliferazione di lingue può creare un nuovo linguaggio composito ed esercitare una forza centrifuga aperta a un mondo che fatica a rivelarsi.

In questa diaspora il francese conserva il proprio status privilegiato di patria ideale per una classe che ha perso i punti di riferimento, sconquassati dal disordine e dall'azzeramento portato dalla guerra: per un Malaparte «romanziere storiografo» e «affrescatore di tempi» ${ }^{30}$ il francese connota di un colore storico l'identità europea messa in crisi e minacciata nel suo stadio finale, ultimo bagliore di un'aristocrazia dispersa. Quell'«"aura" e "clima" di Settecento francese»" che Pancrazi respirava nelle prime novelle di Malaparte continua a circolare nelle opere più mature per quella capacità di ritrarre una nobiltà che sembra immobile nei fasti di una Versailles lontana: «la tecnica stilistica dell'effige è quella della scuola di Saint-Simon-Chateaubriand $\gg^{32}$ e Malaparte rievoca quel mondo scomparso ma lungamente frequentato nelle sue letture se, fra gli autori che lo hanno maggiormente influenzato, ricorderà anche «tous les écrivains qui battirent en brèche tous les illuministes: Montaigne, Voltaire, Montesquieu, Stendhal» ${ }^{33}$. È proprio a una citazione di Montesquieu che è affidato il senso generale di Kaputt, quando conclude la "Storia di un manoscritto" descrivendo i giorni della guerra con un passo dell'Esprit des lois: «Ainsi, dans le temps des fables, après les inondations et les déluges, il sortit de la terre des hommes armés, qui s'exterminèrent $\gg^{34}$. È finito il tempo delle favole, della vecchia Europa, ora sono i tempi crudeli.

Se la crudeltà è una cifra essenziale del libro, vantata dallo stesso autore («Kaputt è un libro crudele» ${ }^{35}$ ), in essa si scontrano due mondi: quello germanico, aggressivo e irrazionale, e quello francese, civile e in pericolo. L'intervento della crudeltà è il segno di un ordine incrinato che stritola le possibilità di esistenza di una civiltà ideale e la relega nel lontano tempo del sogno e della giovinezza. È interessante notare che Malaparte considerava «la crudeltà, questa Musa segreta di molta poesia classica ... ignota ai Francesi $>^{36}$ : rappresentarla non è l'atto di un testimone sadico bensì mettere in mostra una forza estranea. Pure nella mappa degli autori e pittori citati si rivela un criterio non casuale di contrapposizione tra i francesi e i tedeschi: mentre i primi sono oggetto di un piacere estetico vagamente idillico, i secondi portano sempre la durezza e il volto della violenza o della morte. Il preciso disegno di Kaputt si manifesta anche sul piano di una intertestualità che conduce emotivamente e culturalmente a

(29) C. Malaparte, I giovani non sanno scrivere, in «Prospettive», 15 febbraio 1940 e ora in E. RoNCHI SUCKERT, op. cit., vol V (1940-41), pp. 62-66, a p. 63 e 65.

(30) M. Biondi, Malaparte. L'Europa dei vinti, in «La bourse des idées du monde». Malaparte e la Francia. Atti del convegno internazionale di studi su Curzio Malaparte (Prato-Firenze, 8-9 novembre 2007), a cura di M. GRASSI, Firenze, Olschki, 2008, pp. $23-47$, a p. 41.

(31) P. PANCRAzI, Le novelle di Malaparte, in «Corriere della Sera», 6 marzo 1931, citato in L. Martellini, Malaparte narratore, in Nel labirin- to della scrittura, Roma, Salerno Editrice, 1996, pp. 113-170, a p. 133 .

(32) M. BIONDI, Scrittori e miti totalitari: Malaparte, Pratolini, Silone, Firenze, Polistampa, 2002, p. 109

(33) R. GuéRIn, Du côté de chez Malaparte, Bordeaux, Finitude, 2003 [1950], pp. 23-24.

(34) K 431.

(35) K 430.

(36) C. Malaparte, L'ultimo Breton, in «Prospettive», 15 gennaio 1940 , e ora in in E. RONCHI SuCKERT, op. cit., vol V (1940-41), pp. 41-43, a p. 42. 
identificarsi nella Francia aggredita, in una civiltà raffinata che sta scomparendo. Alle tele luminose degli impressionisti, dei fauves e dei cubisti si contrappone una pittura tedesca tradizionalmente lugubre e cupa, da Holbein a Dürer e a Grosz, evocati per descrivere espressionisticamente le mense dei gerarchi nazisti o il paesaggio desolato di un campo di battaglia, dove sembrava che «il bulino dell'incisore si fosse fermato, riposando, un istante e il peso della mano avesse scavato nel rame un segno più profondo» ${ }^{37}$. Così al ricordo di una Parigi gioiosamente art nouveau si contrappone una Berlino tetra e spettrale, dove la vita è diventata la grigia, infernale routine di ingranaggi al servizio dello Stato.

Al mito di Parigi e di una francesità che si diffonde in tutta l'opera di Kaputt fa da riflesso l'avanzata delle armate tedesche e la sciagura dei loro «atti più freddamente, più metodicamente, più scientificamente crudeli» ${ }^{38}$ : non è solo la crudeltà a minacciare Parigi, ma è l'intervento di un ordine nuovo fondato sulla razionalità e la precisione scientifica, dominato dai metodi geometrici e dalle macchine che rimangono come metallici detriti sulle strade dell'Europa: l'esistenza di tutto ciò che è vitale, umano, poetico è messo in crisi non solo dalla morte ma anche e più dal possibile avvento di una nuova era che mescola la barbarie alla razionalità e farà franare nell'oblio l'esuberanza di una Parigi giovane ed elegante.

La messa in discussione della razionalità, unita a un interesse al mondo dei sogni, a una ricostruzione sorretta da nessi a-logici, all'accostamento di immagini anche preziose che tendono alla dilatazione degli spazi e alla loro deformazione, rappresenta la sfida dello scrittore che cerca ancora i valori irrecuperabili dell'arte, la loro sopravvivenza nel caos della guerra: quel «côté de Guermantes» ${ }^{39}$ che anche una cittadina romena può vantare, viene spazzato via dalla furia di metodici esecutori che compiono un orrendo massacro. Il debito di Malaparte nei confronti del Surrealismo ${ }^{40}$ viene qui

(37) K 236.

(38) K 103.

(39) K 593.

(40) È spesso ricordato il merito di Malaparte di aver contribuito alla diffusione del Surrealismo in Italia tramite la rivista «Prospettive» (1939-1943), dove discute del movimento e pubblica, a volte anche nella veste di traduttore, Breton e Éluard (proibito dal regime) oltre a Lautréamont, Ronsard, Scève, Claudel. Un tentativo di ricostruzione si trova in G. ANGELI, Malaparte e il surrealismo, in «La bourse des idées du monde». Malaparte e la Francia, cit., pp. 67-80; tuttavia non condividiamo l'impostazione, che vedrebbe Malaparte un agitatore e rimescolatore funambolico, al contrario a nostro parere Malaparte mostra d'intendere con acuta sensibilità l'importanza storica del movimento (perché oppone «a una tradizione divenuta stile, alla retorica, alla logica ... la necessità di creare nuovi e più propri mezzi d'espressione, d'inventare un nuovo linguaggio poetico, di risolvere i problemi formali della nostra libertà interiore, della nostra fantasia, cioè i problemi dell'espressione», in C. MALAPARTE, Il surrealismo e l'Italia in «Prospettive», 15 gennaio 1940), il rapporto strettissimo tra linguaggio e cose e il lavorio sulla disgregazione della lingua, e la capacità di penetrazione nel subcosciente, che però non vorrebbe affidata alla scrittura automatica né a un metodo razionale ( $\ll i l$ mondo dell'incosciente vuol essere esplorato non da uomini ragionanti, ma da larve addormentate, a occhi chiusi», in C. Malaparte,

\section{L'ultimo Breton, cit.)}

Rosa Galli Pellegrini invece rileva l'importante ruolo svolto da Malaparte nella diffusione del Surrealismo in Italia, per aver fatto di «Prospettive» una tempestiva sede di dibattito tra alcune significative voci critiche o poetiche italiane, con l'aggiunta anche di una prima bibliografia di studi non solo italiani a francesi, ma anche americani e jugoslavi (R. GAlli Pellegrini, Il movimento surrealista e la critica italiana (1925-1944), in «Rivista di Letterature Moderne e Comparate», vol. XXIX, n. 1, marzo 1976, pp. 33-66).

Per quanto riguarda le tracce del Surrealismo nell'opera di Malaparte, Luigi Martellini ha individuato una vaga influenza in alcune novelle (cfr. L. MaRTELLINI, Malaparte narratore, cit.) oltreché in tante pagine di Kaputt, rilevata già da molti critici, così come Enzo Rosario Laforgia ha intravisto le prime contaminazioni surrealiste nei reportages etiopi del 1938 (cfr. E. R. LAFORGIA, Introduzione a C. MALAPARTE, Viaggi in Etiopia e altri scritti africani, a cura di E. R. LAFORGIA, Firenze, Vallecchi, 2007, pp. 7-35). Vogliamo sottolineare che poiché di tracce si tratta, il rapporto tra il movimento francese e la scrittura malapartiana rimane tangenziale e non incisivo, spesso stimolo a forme di scrittura originali e sensuali, non certo allineate all'impianto teorico e pratico del Surrealismo; tuttavia non esitiamo a vedere nel capitolo sesto di Kaputt un esempio di ripensamento della realtà in forme chiaramente oniriche e surreali. 
esibito e allo stesso tempo relegato in una zona della fantasia che si rivela l'unica capace di riportare sulla pagina l'assurdo di una realtà non più intelligibile: la necessità di re-interpretare la realtà con strumenti nuovi porta alla crisi del realismo, allo straniamento che impedisce di riconoscere gli oggetti per quello che sono, all'affollarsi di immagini oniriche che ondeggiano sul reale e sembrano affiorare laddove un mondo fiabesco fa irruzione nel quotidiano voltando le spalle alla tragedia appena consumata. Il narratore riesce con un abile montaggio, un caotico pastiche che va dal surreale all'iperreale ctonio al fiabesco, a smarrire i termini di riferimento, a confondere i nessi logici e ogni intelligibilità razionale, ma con l'oscura coscienza che il massacro c'è stato e non c'è spiegazione: solo dalla deformazione allucinata, talvolta anche grottesca, poteva scaturire una percezione del male così abnormemente limpida e inorridita. In questo naufragio il mondo parigino viene evocato come impotente e devitalizzato, la clarté francese negata e assente: l'elemento strutturale che sembrava garantire unità a questa piccola odissea non può costituirsi come rifugio ideale, arcadia dell'arte e della giovinezza, ma è costretto a regredire in un'illusione che alimenta la scrittura e si offre come chiave e ribaltamento degli eventi narrati. 